\title{
Abordagem teórico-prática da dermatite herpetiforme como a principal manifestação cutânea da doença celíaca: Revisão integrativa
}

\author{
Theoretical-practical approach to herpetiform dermatitis as the main cutaneous manifestation of \\ celiac disease: An integrative review
}

Abordaje teórico-práctico de la dermatitis herpetiforme como principal manifestación cutánea de la enfermedad celíaca: Una revisión integradora

Recebido: 20/04/2021 | Revisado: 03/05/2021 | Aceito: 10/05/2021 | Publicado: 27/05/2021

\author{
Sarah Maria Monteiro Soares Costa de Holanda \\ ORCID: https://orcid.org/0000-0001-9170-107X \\ Centro Universitário Uninovafapi, Brasil \\ E-mail: sarahscholanda@gmail.com \\ Raissa Martins de Oliveira Nunes \\ ORCID: https://orcid.org/0000-0002-4741-2489 \\ Instituto de Educação Superior do Vale do Parnaíba, Brasil \\ E-mail: raiissamartiins@hotmail.com \\ Larissa Alves dos Santos Silva \\ ORCID: https://orcid.org/0000-0001-5767-3140 \\ Universidade Federal do Piauí, Brasil \\ E-mail: larialvessantoss@gmail.com \\ Marianna Mendes de Barros \\ ORCID: https://orcid.org/0000-0003-2657-0055 \\ Centro Universitário Uninovafapi, Brasil \\ E-mail: Mariannamdbarros@gmail.com \\ Pedro Henrique Freitas Silva \\ ORCID: https://orcid.org/0000-0001-7997-119X \\ Centro Universitário Unifacid, Brasil \\ E-mail: pedro.henriquefreitas.silva@hotmail.com \\ Anna Joyce Tajra Assunção \\ ORCID: https://orcid.org/0000-0002-5584-9971 \\ Centro Universitário Unifacid, Brasil \\ E-mail: annajoycetajra@hotmail.com \\ Adhonias Carvalho Moura \\ ORCID: https://orcid.org/0000-0001-7595-4907 \\ Centro Universitário Unifacid, Brasil \\ E-mail: moura.adhonias@gmail.com \\ Evandra Marielly Leite Nogueira Freitas Galvão \\ ORCID: https://orcid.org/0000-0002-9916-9249 \\ Centro Universitário Uninovafapi, Brasil \\ E-mail: evandranogueira@hotmail.com
}

\begin{abstract}
Resumo
Introdução: A dermatite herpetiforme (DH) é uma manifestação benigna, crônica e inflamatória da pele. As lesões são erupções eritêmato- pápulo- vesiculosa, pruriginosas, simétricas, dispostas de forma herpetiforme, tendo afinidade por superfícies extensoras. Há predileção pelo sexo masculino, entre 30 e 40 anos e está relacionada com anormalidades na mucosa do intestino delgado e ao depósito de IgA na derme papilar. Tem origem multifatorial, abrangendo componentes genéticos, ambientais e imunológicos. Objetivos: Analisar a aplicação dos conhecimentos obtidos pela revisão integrativa abordando a dermatite herpetiforme e doença celíaca (DC) para elaboração de um raciocínio clínico frente ao diagnóstico precoce e tratamento efetivo. Metodologia: Artigos científicos e de revisão publicados e referenciados no MEDLINE, LILACS, IBECS, BINACIS, LIS, BRISA/RedTESA e MEdCArib foram consultados, delimitados de 2008 a 2019. Os livros "Tratado de dermatologia" de Belda Júnior e "Dermatologia" de Sampaio e Rivitti também foram incluídos. Resultados: O diagnóstico da DH é clinico, laboratorial e imunológico. A apresentação e atividade de erupção das bolhas é variável. O achado histopatológico clássico é uma fenda subepidérmica com neutrófilos e eosinófilos nas papilas dérmica, com infiltrado inflamatório misto perivesicular. A imunofluorescência direta da pele não envolvida é padrão ouro.O pilar do tratamento envolve uma dieta livre de glúten associado ao uso de medicamentos, sendo observada a remissão incompleta frequente em pacientes que não restringem o glúten. Conclusão: $\mathrm{O}$ conhecimento das manifestações clínicas e subclínicas da DH e DC assim como achados laboratoriais são cruciais ao diagnóstico precoce, corroborando para afastar diagnósticos errôneos, exacerbações ou complicações.

Palavras-chave: Dermatite herpetiforme; Doença celíaca; Lesões cutâneas; Lesões versículobolhosas.
\end{abstract}




\begin{abstract}
Introduction: Dermatitis herpetiformis $(\mathrm{DH})$ is a benign, chronic, inflammatory manifestation of the skin. The lesions are erythemato-papular vesicular eruptions, pruritic, symmetrical, herpetiform in shape, and have an affinity for extensor surfaces. It is male-dominant, between the ages of 30 and 40, and is related to abnormalities of the small intestinal mucosa and to $\operatorname{IgA}$ deposition in the papillary dermis. It has a multifactorial origin, encompassing genetic, environmental, and immunologic components. Objectives: To analyze the application of knowledge obtained by integrative review addressing dermatitis herpetiformis and celiac disease (CD) to develop a clinical rationale for early diagnosis and effective treatment. Methodology: Scientific and review articles published and referenced in MEDLINE, LILACS, IBECS, BINACIS, LIS, BRISA/RedTESA and MEdCArib were consulted, delimited from 2008 to 2019. The books "Tratado de dermatologia" by Belda Júnior and "Dermatologia" by Sampaio and Rivitti were also included. Results: The diagnosis of DH is clinical, laboratory and immunological. The presentation and eruption activity of the blisters is variable. The classic histopathologic finding is a subepidermal cleft with neutrophils and eosinophils in the dermal papillae, with perivesicular mixed inflammatory infiltrate. Direct immunofluorescence of uninvolved skin is the gold standard. The mainstay of treatment involves a gluten-free diet coupled with the use of medications.Incomplete remission is frequently observed in patients who do not restrict gluten. Conclusion: Knowledge of the clinical and subclinical manifestations of DH and CD as well as laboratory findings is crucial for early diagnosis, helping to rule out misdiagnosis, exacerbations, or complications.
\end{abstract}

Keywords: Herpertiformis dermatites; Celiac disease; Skin lesions; Versiculobullous lesions.

\title{
Resumen
}

Introducción: La dermatitis herpetiforme (DH) es una manifestación benigna, crónica e inflamatoria de la piel. Las lesiones son erupciones eritematoso-papular-vesiculares, pruriginosas, simétricas, dispuestas en forma herpetiforme, con afinidad por las superficies extensoras. Existe predilección por el sexo masculino, entre 30 y 40 años, y se relaciona con anomalías en la mucosa del intestino delgado y el depósito de IgA en la dermis papilar. Tiene un origen multifactorial, que engloba componentes genéticos, ambientales e inmunológicos. Objetivos: Analizar la aplicación de los conocimientos obtenidos por la revisión integradora que aborda la dermatitis herpetiforme y la enfermedad celíaca (EC) para la elaboración de un razonamiento clínico ante el diagnóstico precoz y el tratamiento eficaz. Metodología: Se consultaron artículos científicos y de revisión publicados y referenciados en MEDLINE, LILACS, IBECS, BINACIS, LIS, BRISA / RedTESA y MEdCArib, delimitados de 2008 a 2019. Los libros "Tratado de dermatología" de Belda Júnior y "Dermatología" de Sampaio y Rivitti también se incluyeron. Resultados: El diagnóstico de HD es clínico, de laboratorio e inmunológico. La actividad de presentación y erupción de las burbujas es variable. El hallazgo histopatológico clásico es una hendidura subepidérmica con neutrófilos y eosinófilos en las papilas dérmicas, con infiltrado inflamatorio perivesicular mixto. La inmunofluorescencia directa de la piel no afectada es el estándar de oro. El pilar del tratamiento implica una dieta libre de gluten asociada al uso de medicamentos, observándose frecuentes remisiones incompletas en pacientes que no restringen el gluten. Conclusión: El conocimiento de las manifestaciones clínicas y subclínicas de la DH y la EC, así como los hallazgos de laboratorio, son cruciales para el diagnóstico precoz, corroborando para descartar diagnósticos erróneos, exacerbaciones o complicaciones.

Palabras clave: Dermatitis herpetiforme; Enfermedad celíaca; Lesiones cutáneas; Lesiones versículo-ampollosas.

\section{Introdução}

Em 1884 o dermatologista Louis Duhring descreveu a dermatite herpetiforme (DH) na mesma categoria do pênfigo e penfigóide, compondo, dessa forma, a classe de doenças bolhosas. Quatro anos depois, em 1884, foram descritas lesões cutâneas semelhantes, diagnosticadas como “dermatite prurítica polimórfica” por Brocq, que após analisar o relatório de Duhring concluiu que se tratavam da mesma patologia. Em razão disso, a DH também pode ser conhecida como doença de Duhring-Brocq (Mendes et al., 2013).

Civatte, em 1943, diferenciou pênfigo (bolhas intra-epidérmicas), penfigóide e DH (formação de bolhas na zona da membrana basal). No entanto, a associação da DH com a doença celíaca só foi observada por Mards et al., Fry et al. e Suhster et al. nos anos sessenta (Clarindo et al.,2014).

Gjone e Nordoy foram os primeiros a relatar o aumento da incidência de linfoma em pacientes com DH em 1970. Esta percepção foi confirmada por décadas, no entanto, em 2008, um estudo realizado com 846 pacientes e feito por Lewis et. al., contradisse essa relação, uma vez que, não encontraram aumento do risco de mortalidade nesses pacientes (Mendes et al., 2013).

A doença de Duhring- Brocq é caracterizada por uma manifestação benigna, crônica e inflamatória da pele. As lesões seguem um padrão de erupção eritêmato- pápulo- vesiculosa, pruriginosas, simétricas e dispostas de forma herpetiforme. Essas 
lesões costumam ser generalizadas e ter afinidade por superfícies extensoras, como joelhos e cotovelos. Couro cabeludo, região cervical, membros, cintura escapular, face e nádegas também podem ser acometidas (Castro et al., 2014).

Encontrada na grande maioria em homens, a DH pode acometer crianças e idosos, no entanto, possui uma maior incidência na faixa etária entre 30 e 40 anos. Ela está relacionada com anormalidades na mucosa do intestino delgado e ao depósito de IgA na derme papilar. Nos pacientes com DC, uma média de 15 a 25\% deles apresentam DH e destes, menos de 10\% apresentam um quadro evidente de DC com diarreia, desconforto abdominal, perda de peso e má absorção de nutrientes (Rivitti, 2018).

Além disso, é notória a maior prevalência da DH em brancos, se comparada com negros e asiáticos, visto na África há um baixo consumo de trigo. Em contrapartida, há uma maior ingestão de trigo no EUA e no norte da Europa, de forma a existir uma maior prevalência da doença (Reunal, Salmi \& Hervonen, 2015).

A doença é conhecida por um desenvolvimento de origem multifatorial, no qual tem relação com componentes genéticos, ambientais e imunológicos. De fator ambiental, observa-se a exposição ao glúten em períodos que agravam a dermatose, de cunho genético há predisposição pela sua relação com os HLA DQ2 (90\%) e DQ8 (10\%), é importante ressaltar que a DC e a DH compartilham do mesmo background genético, tendo forte associação com HLA-DQ2 (Hervonen et al, 2016). Além disso, imunologicamente a DH está relacionada ao auto- antígeno transglutaminase epidérmica (eTG) e a antiglutaminase tecidual (TTG) (Coutinho et al., 2014).

Além da patogênese multifatorial, observa-se estreita relação da DH com a doença celíaca (DC) em que ambas demonstram intolerância ao glúten, mediação imunológica pelos anticorpos $\operatorname{Ig} \mathrm{A}$ e em média 75\% dos indivíduos com DH possuem atrofia das vilosidades e inflamação da mucosa do intestino delgado, como observada na DC. Outro fator pode ser percebido, na resolução das lesões das duas patologias após a retirada do glúten. Com isso, podemos citar a dermatite herpetiforme como apresentação cutânea da doença celíaca (Costin et al., 2019).

A DH é a manifestação cutânea relacionada à sensibilidade ao glúten, além de ser a manifestação extra-intestinal da DC (Sgnaolin et al., 2014). Diante disso, o diagnóstico correto e precoce é de suma importância para o tratamento e resolução do quadro clínico. Por conta dos sinais e sintomas serem confundidos com outras patologias, é importante fazer uma consulta integral. Doenças como penfigóide bolhoso, eritema polimorfo, dermatose por IgA linear, eczema atópico fazem diagnóstico diferencial com a DH (Coutinho et al., 2014).

O diagnóstico da dermatite herpetiforme, baseia-se na clínica, imunoflorescência direta (IFD), histologia cutânea e sorologia. A imunoflorescência direta ou gold standard retrata os depósitos de IgA na junção dermoepidérmica, sendo patognomônico da DH. A análise histológica típica das lesões cutâneas mostra vesículas ou bolhas na junção dermoepidérmica com depósitos de neutrófilos e, também, eosinófilos nas zonas papilares na biopsia das erupções. Já a sorologia, vão quantificar anticorpos antitransglutaminase (tTGA) e antiendomísio (EMA) da classe IgA, presentes na DH e DC (Sgnaolin et al., 2014).

A sorologia além de ser meio de diagnóstico, também pode ser usada para monitoramento da resposta terapêutica, no qual a ausência de títulos de tTGA e EMA representam a ausência da patologia, consequentemente, o cumprimento do tratamento. Além disso, vale ressaltar que a biópsia intestinal dos pacientes com DH vão apresentar distorções iguais na DC, como a presença de infiltrativo subtil com atrofia parcial ou sem atrofia das vilosidades, mas sendo lesões mais rápidas e de distribuição diferente (Farinha et al., 2018).

Após o diagnóstico da DH é mandatório o tratamento com a restrição do glúten nas refeições. Apesar de não existir medicamentos curativos, o uso da dapsona concomitantemente a retirada do glúten mostrou altos índices de controle e melhora do quadro de prurido e erupções cutâneas. Apesar da dapsona oral ser a primeira linha do tratamento farmacológico, antes de começar seu uso deve-se confirmar níveis normais de glicose-6- fosfato desidrogenase (Costin et al., 2019). 
Outros fármacos de $2^{\mathrm{a}}$ geração podem ser utilizados no tratamento, como a sulfalazina e sulfametoxipridazina em doses de 1-2g/ dia e 0,25-1,5g/ dia, respectivamente, quando a dapsona não for possível por apresentar efeitos adversos superiores aos benefícios (Coutinho et al., 2014).

A prevenção de complicações futuras é essencial, a mesma é realizada com a restrição ao glúten, a fim de evitar linfomas do intestino delgado (Farinha et al., 2018). Após o início do tratamento nutricional, pode levar até 2 anos para resolução do quadro intestinal. Com o uso, concomitante, da dapsona em doses de $1 \mathrm{mg} / \mathrm{kg} / \mathrm{dia}$ no adulto, resulta em melhoras do quadro cutâneo em até 48-72 h (Coutinho et al., 2014).

Objetiva-se com o presente estudo analisar a aplicação dos conhecimentos obtidos pela revisão integrativa abordando a dermatite herpetiforme e doença celíaca em prol da elaboração de um raciocínio clínico no ambulatório de dermatologia frente ao diagnóstico precoce e consequente tratamento efetivo.

\section{Metodologia}

A revisão integrativa da literatura decorre de um estudo com análise ampla de dados feitos a partir de literaturas secundárias, através do delineamento bibliográfico e tendo como parâmetro a experiência vivida no cotidiano das autoras, resultando na realização do presente estudo (Souza, Silva \& Carvalho, 2010). De forma que, o objetivo desse estudo é proporcionar uma síntese dos conhecimentos mais atuais e relevantes acerca da dermatite herpetiforme e sua relação com a doença celíaca, além da aplicabilidade teórico-prática.

Inicialmente, realizou-se uma primeira avaliação, tendo como base análise de artigos e literaturas. Em seguida, foram descartados aqueles trabalhos que não preencheram os critérios de inclusão e exclusão pré-estabelecidos para a elaboração desse referido estudo. Portanto, o critério de inclusão usado foi artigos e literaturas em língua nacional e estrangeira (inglesa e francesa) que abordam as manifestações cutâneas da doença celíaca, em específico a dermatite herpetiforme. Já com o critério de exclusão, foram descartados os artigos e literaturas que não abordavam a manifestação dermatológica na doença celíaca.

Ademais, foram usados como instrumento de coleta de dados artigos nas línguas portuguesa, inglesa e francesa encontrados nas bases de dados: MEDLINE, LILACS, IBECS, BINACIS, LIS, BRISA/RedTESA e MEdCArib. Para isso, quatro descritores foram usados: dermatite herpetiforme and doença celíaca and lesões cutâneas or lesões versículobolhosas. Além disso, o período de pesquisa de base de dados se delimitou a partir do ano de 2008 a 2019.

A partir da combinação dos descritores foram obtidos 582 estudos. Em uma primeira avaliação por meio dos resumos, foi verificado que 548 fugiam ao recorte temporal e/ou não abordavam a temática de forma a contribuir para a revisão integrativa. Sendo assim, 34 artigos foram analisados, e desses 19 foram selecionados e incluídos na versão final dessa revisão integrativa de literatura. Além disso, os livros "Tratado de dermatologia" do autor Belda Júnior e "Dermatologia" de Sampaio e Rivitti também foram incluídos. 
Figura 1 - Fluxograma da coleta de dados.

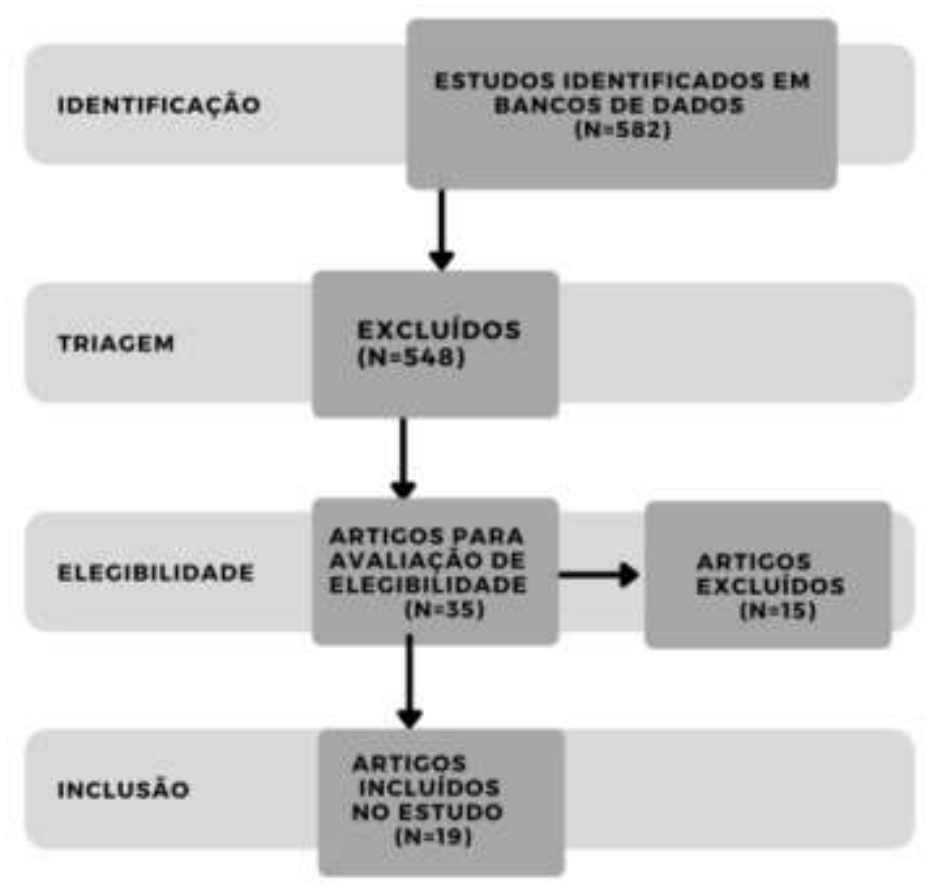

Fonte: Autores.

\section{Resultados e Discussão}

Nas últimas décadas a média da idade dos pacientes que são acometidos pela DH aumentou significativamente, variando em cada região investigada. Estima-se a média de idade de 40 a 50 anos e a proporção entre homens e mulheres varia de 2:1 em grandes estudos realizados na Europa e na América do Norte, além disso, a doença é rara na infância (Reunala et al., 2018).

Os estudos mostram que devido à cultura de países como Europa e EUA há uma alta ingestão de trigo, assim tornou-se notório a maior prevalência da DH e da DC entre brancos em determinadas regiões do mundo. Além disso, a razão de prevalência da DH para DC é de 1:8 na Finlândia e no Reino Unido, em alguns casos a DH é a manifestação cutânea da doença celíaca (Reunala et al., 2018).

A DH caracteriza-se por lesões polimórficas, eritemato- descamativas, pruriginosas e com predileção por membros extensores, sendo eles os mais comuns cotovelos e joelhos. Entretanto, devemos ressaltar que há relatos de manifestações em sulcos infra-nadegueiros, punhos, couro cabeludo, face, cintura escapular, região cervical e rara na mucosa oral (Calado et al., 2008).

Em pacientes com DH, acredita-se que a formação das placas cutâneas pruriginosas e descamativas, características da doença, ocorrem por conta do acúmulo na derme de imunocomplexos circulantes, gerando uma quimiotaxia e ativando neutrófilos, mediante a isso há formação das lesões na pele (Bonciolini et al., 2018).

Contudo, a apresentação e a atividade de erupção das bolhas oscilam muito de acordo com o paciente. Mas observou que a remissão incompleta é frequente em pacientes que não aderem ao tratamento com restrições ao glúten (Reunala, Salmi \& Hervonen, 2015).

A imunopatogênese da DH e DC revelaram uma estreita relação de ambas com os alelos que contribuem para o halótipo HLA- DQ2 ou HLA- DQ8, presentes até em gêmeos monozigóticos. Além disso, uma hipótese a ser considerada é que a DH inicia com uma DC latente e evolui com a deposição de imunocomplexo de anticorpos IgA transglutaminase epidérmica (TG3) com uma avidez alta, em conjunto com a enzima TG3 na derme papilar (Reunala et al., 2018). 
Vale ressaltar que anticorpos de $\operatorname{IgA}$ são produzidos no intestino em complexos imunes glúten- antiglúten. Em conseqüência disso, o complexo de IgA são retidos na pele em resultado da reação cruzada com os anticorpos de reticulina depositados (Reunala, Salmi \& Hervonen, 2015).

A erupção única das bolhas no paciente com DH mostrou ter relações com fatores locais. A força mecânica pode ativar a TG3 em agregados dérmicos, igual à ativação de transglutaminase tecidual (TG2) nas paredes vasculares. Com isso, irá resultar na liberação de fibrinogênio que é um fator de coagulação e uma proteína inflamatória capaz de recrutar células T, neutrófilos e macrófagos. Desse modo, todos os fatores alinhados serão para desenvolver as lesões na DH (Reunala, Salmi \& Hervonen, 2015).

A prevalência de HLA DQ2 e DQ8 é a mesma em DC E DH, corroborando com o conceito de que DH é uma manifestação dermatológica da DC. 90\% dos pacientes com DH expressam HLA DQ2, os outros DQ8 e os pacientes sem os dois tipos são extremamente raros. Observa-se que a DH como a DC é familiar, pois 10-15\% dos familiares de com DH apresentam DC ou DH (Kotze, 2013).

De acordo com Kotze (2013), o diagnóstico da DH é clinico, laboratorial e imunológico. O achado histopatológico clássico na DH é uma fenda subepidérmica com neutrófilos e alguns eosinófilos nas pontas das papilas dérmicas geralmente com infiltrado inflamatório misto perivesicular, porém os pacientes com lesões pruringinosas escoreadas os achados histológicos não vão confirmar a DH, provavelmente pelo erro de local da biópsia. A imunofluorescência direta da pele não envolvida coletada no local perilesional de aparência normal é padrão ouro, visto que, nessa área apresenta uma maior deposição de IgA do que na pele não lesionada ou lesionada.

Ao analisar a histologia é possível observar um infiltrado de neutrófilos polinucleados (PNN) no topo das papilas dérmicas, porém a sua produção não se dá no mesmo local. Esse infiltrado pode estar associado aos descolamentos subepidérmicos que podem ocorrer em fases mais posteriores e exacerbações da doença. A imunofluorescência direta mostra a deposição de anticorpos IgA granular no topo das papilas dérmicas, e mesmo sob efeito do tratamento esses anticorpos podem persistir no local. O principal autoanticorpo na DH é o IgA anti-TGE, porém o mecanismo que explica a avidez desses anticorpos às células dérmicas ainda não é totalmente elucidado, algumas hipóteses falam da existência de complexos imunes circulantes que explicam a colocação desses autoanticorpos na derme. Esse fato pode contribuir para o aparecimento dessa doença de forma paradoxal em pacientes com deficiência parcial de IgA, assim como, justificaria a quantidade reduzida de pacientes com DC que desenvolvem DH (Doffoel-Hantza et al., 2008).

O teste sorológico de primeira escolha quando houver suspeita de DH e DC é o ELISA, no qual testa anticorpos TG2 de classe IgA. O anticorpo antigliadina não é mais usado devido a sua falta de especificidade, já os anticorpos endomisiais tem confiabilidade comparável para DH e DC como os anticorpos TG2, porém esse teste é mais dificultoso e subjetivo na interpretação. Os anticorpos séricos TG2 mostraram correlação com o grau de dano à mucosa intestinal na HD, sendo menos prevalentes na HD do que na DC. Assim, a presença dos anticorpos TG2 apoia o diagnóstico, mas na sua ausência não exclui DH. Ademais, além da pele, já foi documentado que a maior parte dos pacientes com DH tem auto anticorpos circulantes contra TG3, este sendo o auto antígeno da DH, no entanto, podem existir ocasionalmente TG3 no soro de pacientes com DC sem quaisquer sintomas cutâneos. Dessa forma, a especificidade de anticorpos TG3 para DH ainda não é confirmada, sem usabilidade desse teste na pratica clinica atual (Salmi, 2019).

É válido ressaltar que a DH pode ser confundida com lúpus eritematoso sistêmico bolhoso, eczema atópico, dermatite alérgica de contato, sarcoptose, escoriações neuróticas, urticária papulosa e doença bolhosa autoimune. É extremamente importante realizar um diagnóstico diferencial, de forma a corroborar com o tratamento adequado e reconhecimento precoce, evitando a exacerbação dos sintomas e a utilização de fármacos não eficazes ou contraindicados para essa afecção (Farinha, 2018). 
Dentre os diagnósticos diferenciais expressivos da DH, destaca-se os lúpus eritematoso sistêmico bolhoso (LESB), que é um subtipo raro dos lúpus eritematosos sistêmicos (LES), de forma que os estudos mostram menos de 5\% dos pacientes com LES apresentando lesões bolhosas. Sendo o LESB uma desordem bolhosa adquirida, causada por anticorpos contra o colágeno tipo VII, logo, distinta clínica e histopatologicamente do LES (Tincopa et al., 2010).

Como características clínicas, o LESB apresenta erupções cutâneas vésico-bolhosas disseminadas, restritas ou não a áreas fotoexpostas. De forma que essas bolhas podem ser grandes e tensas, aparentando-se ao penfigoide bolhoso, ou pequenas e agrupadas, semelhantes à dermatite herpetiforme. Existe predileção pelo tronco e região supraclavicular, podendo comprometer mucosas. Na evolução a hiperpigmentação residual e formação de cicatrizes ou milium, podem ocorrer, além de manifestações pruriginosas. $\mathrm{Na}$ análise histopatológica, observam-se bolha subepidérmica com microabscessos de neutrófilos nas papilas dérmicas, infiltrado inflamatório linfomononuclear perivascular ou pode ocorrer vasculite leucocitoclástica. Na imunofluorescência direta, ocorre depósito linear ou granular de IgG, IgA e C3 na zona de membrana basal (Obermoser \& Pascual, 2010).

A DH e o LESB se assemelham clínica e histopatologicamente, no entanto, na primeira há a presença de autoanticorpos marcadores da doença glúten-sensível: antigliadina, antiendomísio e antitransglutaminase tecidual. Somado ao fato de que na imunofluorescência direta ocorre depósito de IgA nas formas granulares, fibrilares ou pontilhadas, concentrada nas papilas dérmicas e pela zona de membrana basal. Outro aspecto é que LESB apresenta, além de $\operatorname{IgA}$, também $\operatorname{IgG}$ e IgM na zona de membrana basal, podendo ser fator para diferenciá-lo da DH, que apresenta somente depósito de IgA (Barbosa et al., 2011).

Pacientes com DH têm comprovadamente risco aumentado para desenvolver linfoma. Estudos puderam demonstrar o efeito protetor da dieta sem glúten quanto a essa complicação, sendo que o desenvolvimento do linfoma, principalmente no trato gastrointestinal e nódulos linfáticos associados, pode ser relacionado ao estímulo a longo prazo dos linfócitos locais, secundário à exposição ao glúten (Clarindo et al., 2014).

O objetivo do tratamento reside em controlar os sintomas dermatológicos e digestivos, quando presentes. O pilar do tratamento envolve a adesão de uma dieta livre de glúten associado ao uso de medicamentos, isso acontece por que após um variável período de tempo, muitos pacientes que fazem uso do tratamento medicamentoso experimentam a redução e em alguns casos a descontinuação medicamentosa. Dessa forma, o seguimento de uma dieta livre de glúten, além de ser, essencial para a melhora dos sintomas gastrointestinais. Também é de crucial importância para a manutenção, em longo prazo, do quadro dermatológico (Vale et al., 2019).

A dieta livre de glúten, normalmente, promove melhora dos sintomas gastrointestinais muito mais cedo do que a melhora dos sintomas cutâneos. Isso ocorre, porque os depósitos de IgA podem levar vários anos até serem completamente eliminados da junção dermoepidérmica. Isso é comprovado pelo fato de que a melhora da DH pode levar até dois anos para ocorrer se for usada apenas a dieta como forma de terapia. Os medicamentos de escolha, por outro lado, promovem uma melhora rápida do quadro dermatológico. Somado a isso, evidências apontam que após 5 anos de dieta alimentar, ocorre um efeito protetor contra o desenvolvimento de linfomas no trato gastrointestinal e a regeneração da mucosa intestinal, promovendo uma melhora no quadro de má absorção em pacientes que apresentam esse sintoma. É importante ressaltar que esse quadro de má absorção pode influenciar no desenvolvimento de crianças afetas pela doença, fazendo-se o acompanhamento das mesmas e a avaliação de suas curvas de crescimento (Clarindo et al., 2014).

Apesar dos seus inúmeros benefícios, uma rígida dieta livre de glúten não é fácil de ser mantida, além de possuir um maior custo financeiro, se comparada com uma dieta sem restrições, seus alimentos nem sempre são fáceis de serem encontrado no mercado. Nessa dieta, alimentos derivados do trigo, cevada, centeio e malte devem ser descartados. O consumo de aveia é permitido, no entanto, deve-se ter cautela, pois a maioria dos produtos de aveia no mercado estão contaminados com glúten. Os pacientes devem ser orientados a sempre observar os rótulos de alimentos processados, aditivos alimentares e farmacêuticos e 
não ingerirem alimentos com ingredientes desconhecidos, com o intuito de evitarem produtos com glúten. Em razão dos sintomas gastrointestinais estarem ausentes ou muito leves, na maioria dos casos, e da condição dermatológica ser rapidamente solucionada por medicamentos, é difícil convencer os pacientes a aderirem uma dieta rígida, levando em consideração o seu custo e as limitações sociais associadas. Por esse motivo é importante que o paciente receba auxílio de um nutricionista e participe de grupos de apoio para adquirem um maior conhecimento sobre a sua condição (Vale et al., 2019).

Apesar de muito eficaz, a dieta livre de glúten precisa de um período maior de tempo (de meses a anos) para promover melhoras no quadro dermatológico. Dessa forma, a dapsona, é a droga de primeira escolha para o tratamento da DH, uma vez que, alivia o prurido em horas e melhora as lesões cutâneas em dias. Apesar disso, a droga não possui efeito na doença intestinal ou na redução de risco de linfoma. A dapsona atua inibindo a quimiotaxia de neutrófilos, a liberação de leucotrienos e prostaglandinas e reduzindo o dano tecidual mediado por neutrófilos e eosinófilos (Clarindo et al., 2014).

A dosagem de início deve ser de 50mg/dia com aumento gradativo até 200mg/dia (a depender da tolerância e da resolução do quadro dermatológico). A dosagem de $0,5-1 \mathrm{mg} / \mathrm{kg} / \mathrm{dia}$ é geralmente eficaz para o controle do prurido e prevenção do aparecimento de novas lesões, para crianças é recomendada a dose de $1-2 \mathrm{mg} / \mathrm{kg} / \mathrm{dia}$. A menor dose necessária para manter a remissão deve ser estabelecida e depois interrompida quando forem obtidos os benefícios da dieta, o que ocorre, normalmente, após 1-2 anos (Vale et al., 2019). No entanto, uma pequena parcela dos pacientes com DH possui erupção cutânea de DH ativa apesar de uma adesão à uma dieta livre de glúten estrita por vários anos, a essa condição denominamos DH refratária, ela demonstrou ocorrer em aproximadamente $2 \%$ dos pacientes com DH (Salmi, 2019).

A principal diferença entre a DH refratária e a DC refratária é o fato de que pacientes com DH refratária possuem resposta à dieta livre de glúten na mucosa do intestino delgado e a presença de anticorpos circulantes do tipo celíaco. Dessa forma, uma dar principais características da DH refratária é a presença de depósitos de IgA, sendo um marcador da atividade cutânea contínua (Hervonen et al., 2016).

Alguns cuidados devem ser tomados antes do início da terapia com dapsona, sendo eles, exames laboratoriais, incluindo glicose-6-fosfato desidrogenase (G6PD), hemograma completo, contagem de reticulócitos, enzimas hepáticas, bilirrubina, creatinina e urinálise. Esses exames devem ser repetidos a cada 2 semanas nos primeiros 3 meses de tratamento e depois a cada 3 meses (Vale et al., 2019).

Os efeitos adversos à dapsona podem ser classificados como, tóxicos (metahemoglobinemia, anemia hemolítica, sendo a metahemaglobinemia mais frequente e insidiosa) ou idiossincráticas (síndrome de hipersensibilidade da dapsona; mal-estar geral, erupção exantemática, fotosensibilidade, efeitos neurológicos, nefropatia, hipotireoidismo, efeitos gastrointestinais). Em decorrência disso, é de suma importância fazer o acompanhamento das funções renais e hepáticas. Temos a deficiência de glicose-6-fosfato desidrogenase (G6PD) como contraindicação absoluta para o uso da dapsona, em razão do alto risco de hemólise; embora pacientes sem essa deficiência também possa desenvolver essa complicação. A administração diária de 800 unidades de vitamina A por 4 semanas pode ajudar a prevenir esse efeito colateral (Kotze, 2013)

Quando não é possível o uso da Dapsona, seja por seus efeitos adversos, seja pela falta de resposta (menos frequentemente) a Sulfassalazina é a droga de segunda linha. A eficácia da Sulfassalazina é menos previsível, se comparada com a dapsona, devido à sua absorção variável. Sua dose usual recomendada é de 1-2 g/d, mas pode ser necessário até 4g/d. Sintomas digestivos, náuseas, vômitos e anorexia estão dentro as reações adversas mais comuns, associadas ao seu uso e podem ser evitadas com o uso de comprimidos com revestimento entérico. Dentre as menos comuns estão a anemia hemolítica, reações de hipersensibilidade, proteinúria e criatalúria. No entanto, como forma de monitorar os pacientes, deve ser realizado hemograma completo e urinálise, antes do início do tratamento e mensalmente nos primeiros três meses e a cada 6 meses a partir de então. Drogas como colchicina, ciclosporina, heparina, tetraciclina e nicotinamida também demonstram efeitos benéficos de outras 
drogas alternativas na DH. Imunossupressores, como metotrexato, azatioprina e micofenolato mofetil, podem ser indicados em casos refratários (Vale et al., 2019).

\section{Conclusão}

Concluímos que é de suma importância a realização precoce do diagnóstico, a partir das manifestações clínicas, subclínicas e da técnica mais adequada ao paciente. Dessa forma, é possível mitigar as ocorrências de diagnósticos errôneos, exacerbações da doença e futuras complicações. É importante salientar, ainda, que estudos futuros sobre as manifestações cutâneas da doença celíaca devem ser instigados, vista a ampla forma de manifestação das lesões e a importância do seu reconhecimento para o melhor segmento prognóstico do paciente. A revisão de literatura realizada facilita visualizar de forma abrangente tais manifestações, assim otimizando o diagnóstico e tratamento da doença.

\section{Referências}

Barbosa, W. S., Rodarte, C. M., Guerra, J. G., Maciel, V. G., Fleury Júnior, L. F., \& Costa, M. B. (2011). Lúpus eritematoso sistêmico bolhoso: diagnóstico diferencial com dermatite herpetiformeBullous systemic lupus erythematosus: differential diagnosis with dermatitis herpetiformis. Anais Brasileiros de Dermatologia, 86(4, Suppl. 1), 92-95. https://dx.doi.org/10.1590/S0365-05962011000700024

Belda Junior, W., Di Chiacchio, N., Criado, P. R. (2015). Tratado de dermatologia (2nd ed.) Editora Atheneu. ISBN 853880538X.

Bonciolini, V., Antiga, E., Bianchi, B., Del Bianco, E., Ninci, A., Maio, V., Pimpinelli, N., \& Caproni, M. (2019). Granular IgA Deposits in the Skin of Patients with Coeliac Disease: Is it Always Dermatitis Herpetiformis?. Acta dermato-venereologica, 99(1), 78-83. https://doi.org/10.2340/00015555-3001

Calado, G., Loureiro, G., Ribeiro, C., Machado, D., Tavares, B., \& Chieira, C. (2008). Dermatite herpetiforme como manifestação inicial de doenças celíaca durante a gravidez: caso clínico. Revista Portuguesa de Imunoalergologia, 16(6), 573-582. http://hdl.handle.net/10400.4/1262

Castro, C. G. C., Cunha, C. G., de Lima, C. M., dos Reis, D. Z., Sirio, D. M. B., Bernardes Filho, F., \& Plata, G. T. (2014). Dermatite herpetiforme com exacerbação clínica após interrupção do uso de anovulatório oral. Journal of the Portuguese Society of Dermatology and Venereology, 72(2), 219-222. https://doi.org/10.29021/spdv.72.2.258

Clarindo, M. V., Possebon, A. T., Soligo, E. M., Uyeda, H., Ruaro, R. T., \& Empinotti, J. C. (2014). Dermatitis herpetiformis: pathophysiology, clinical presentation, diagnosis and treatment. Anais brasileiros de dermatologia, 89(6), 865-877. https://doi.org/10.1590/abd1806-4841.20142966

Costin, A., António, A. M., Furtado, C., \& Bártolo, E. (2019). Dermatitis herpetiformis in an adolescent patient. Anais brasileiros de dermatologia, 94(4), 495496. https://doi.org/10.1590/abd1806-4841.20198227

Coutinho, I., Jerónimo, M., Brinca, A., Tellechea, Ó., Moreno, A., \& Figueiredo, A. (2014). Dermatite herpetiforme em idade pediátrica-um diagnóstico a ter em conta. Journal of the Portuguese Society of Dermatology and Venereology, 72(3), 371-375. https://doi.org/10.29021/spdv.72.3.281

Doffoel-Hantz, V., Cogne, M., Sparsa, A., Bonnetblanc, J. M., Drouet, M., Bedane, C. (2008). Physiopathologie de la dermatite herpétiforme. In: Annales de dermatologie et de vénéréologie. Elsevier Masson, 135(11), 789-790. https://pascal-francis.inist.fr/vibad/index.php?action=getRecordDetail\&idt=20810482

Farinha, S., Jordão, F., Tomaz, E., \& Inácio, F. (2018). Dermatite herpetiforme. Caso clínico. Revista Portuguesa de Imunoalergologia, 26(2), 127-131. https://www.researchgate.net/profile/Sofia-Farinha-

2/publication/326941464_Dermatitis_herpetiformis_Clinical_case/links/5d91df0a299bf10cff1a56f0/Dermatitis-herpetiformis-Clinical-case.pdf

Hervonen, K., Salmi, T. T., Ilus, T., Paasikivi, K., Vornanen, M., Laurila, K., Lindfors, K., Viiri, K., Saavalainen, P., Collin, P., Kaukinen, K., \& Reunala, T. (2016). Dermatitis Herpetiformis Refractory to Gluten-free Dietary Treatment. Acta dermato-venereologica, 96(1), 82-86. https://doi.org/10.2340/000155552184.

Kotze, L. M. (2013). Dermatitis herpetiformis, the celiac disease of the skin!. Arquivos de gastroenterologia, 50(3), 231-235. https://doi.org/10.1590/S000428032013000200041

Mendes, F. B., Hissa-Elian, A., Abreu, M. A., \& Gonçalves, V. S. (2013). Review: dermatitis herpetiformis. Anais brasileiros de dermatologia, 88(4), 594-599. https://doi.org/10.1590/abd1806-4841.20131775

Obermoser, G., \& Pascual, V. (2010). The interferon- $\alpha$ signature of systemic lupus erythematosus. Lupus, 19(9), 1012-1019. https://doi.org/10.1177/0961203310371161

Reunala, T., Salmi, T. T., \& Hervonen, K. (2015). Dermatitis herpetiformis: pathognomonic transglutaminase IgA deposits in the skin and excellent prognosis on a gluten-free diet. Acta dermato-venereologica, 95(8), 917-922. https://doi.org/10.2340/00015555-2162

Reunala, T., Salmi, T. T., Hervonen, K., Kaukinen, K., \& Collin, P. (2018). Dermatitis Herpetiformis: A Common Extraintestinal Manifestation of Coeliac Disease. Nutrients, 10(5), 602. https://doi.org/10.3390/nu10050602

Rivitti, E. A. (2018). Dermatologia de Sampaio e Rivitti (4nd ed.). Artes medicas.

Salmi T. T. (2019). Dermatitis herpetiformis. Clinical and experimental dermatology, 44(7), 728-731. https://doi.org/10.1111/ced.13992 
Research, Society and Development, v. 10, n. 6, e18110615276, 2021

(CC BY 4.0) | ISSN 2525-3409 | DOI: http://dx.doi.org/10.33448/rsd-v10i6.15276

Sgnaolin, V., Baldisserotto, V., Stein, RT, \& Epifanio, M. (2014). Dermatite herpetiforme como manifestação única da doença celíaca: relato de caso e revisão da literatura. Scientia Medica, 23 (4), 250-254. https://doi.org/10.15448/1980-6108.2013.4.15468

Souza, M. T. D., Silva, M. D. D., \& Carvalho, R. D. (2010). Revisão integrativa: o que é e como fazer. Einstein (São Paulo), 8(1), 102-106. http://dx.doi.org/10.1590/s1679-45082010rw1134

Tincopa, M., Puttgen, K. B., Sule, S., Cohen, B. A., \& Gerstenblith, M. R. (2010). Bullous lupus: an unusual initial presentation of systemic lupus erythematosus in an adolescent girl. Pediatric dermatology, 27(4), 373-376. https://doi.org/10.1111/j.1525-1470.2010.01179.x

Vale, E., Dimatos, O. C., Porro, A. M., \& Santi, C. G. (2019). Consensus on the treatment of autoimmune bullous dermatoses: dermatitis herpetiformis and linear IgA bullous dermatosis - Brazilian Society of Dermatology. Anais brasileiros de dermatologia, 94(2 Suppl 1), 48-55. https://doi.org/10.1590/abd18064841.2019940208 